\title{
Kepler's cosmos
}

\section{Copernicus's system of the Universe was revolutionary but his method of representing it on paper was anything but. It was left to Kepler to apply Renaissance techniques of spatial visualization to make the theory come alive.}

\section{Martin Kemp}

$\mathrm{N}$ icolaus Copernicus's programme to purge the Ptolemaic model of the Universe of its growing burden of disfiguring complications was driven not least by what we would call aesthetic considerations. The architecture of his heliocentric system reinstated the geometrical integrity that the Greek astronomers had sought: "At rest... in the middle of everything is the sun. For in this most beautiful temple, who would place this lamp in another or better position than that from which it can light up the whole thing at the same time?"

Although Copernicus's "temple" obeyed the principles of harmonically unified design advocated in the Renaissance, his conventionally diagrammatic representation of his scheme in 1543 as a flat series of concentric circles did not avail itself of the new spatial vision inherent in the buildings and paintings of the Renaissance masters. It was more than 50 years later that Johannes Kepler, fervent Copernican and Platonist, allied the new visual forms with the new astronomical vision.

Among the many testimonies to Kepler's extraordinary powers of spatial visualization, none is more remarkable than the great cosmological model he illustrated in a foldout plate in his Mysterium Cosmographicum of 1596 . We know how the scheme came to be envisaged. He tells how, when he was teaching his "students the way Great Conjunctions jump eight signs at a time", he drew "many triangles, or quasi triangles, in the same circle".

\section{It began to dawn on him that the distances between the orbits of the planets could be explained if the ratios between successive orbits were designed to be equivalent to the spheres successively circumscribed around and inscribed within the five 'Platonic solids' (the regular polyhedra), nested one inside the other.}

Kepler envisaged that his Platonic machine, in its most ambitious form, would be driven by clockwork and that its hollow armature would be filled by alcoholic beverages of a suitable kind.It was, after all, a courtly creation, dedicated to Duke Frederick of Württemberg, who literally acted as a protector for Kepler as a Copernican 'heretic'. The form of visualization and representation in the model stood in direct line of succession from the form of spatial illustration invented by Leonardo da Vinci for

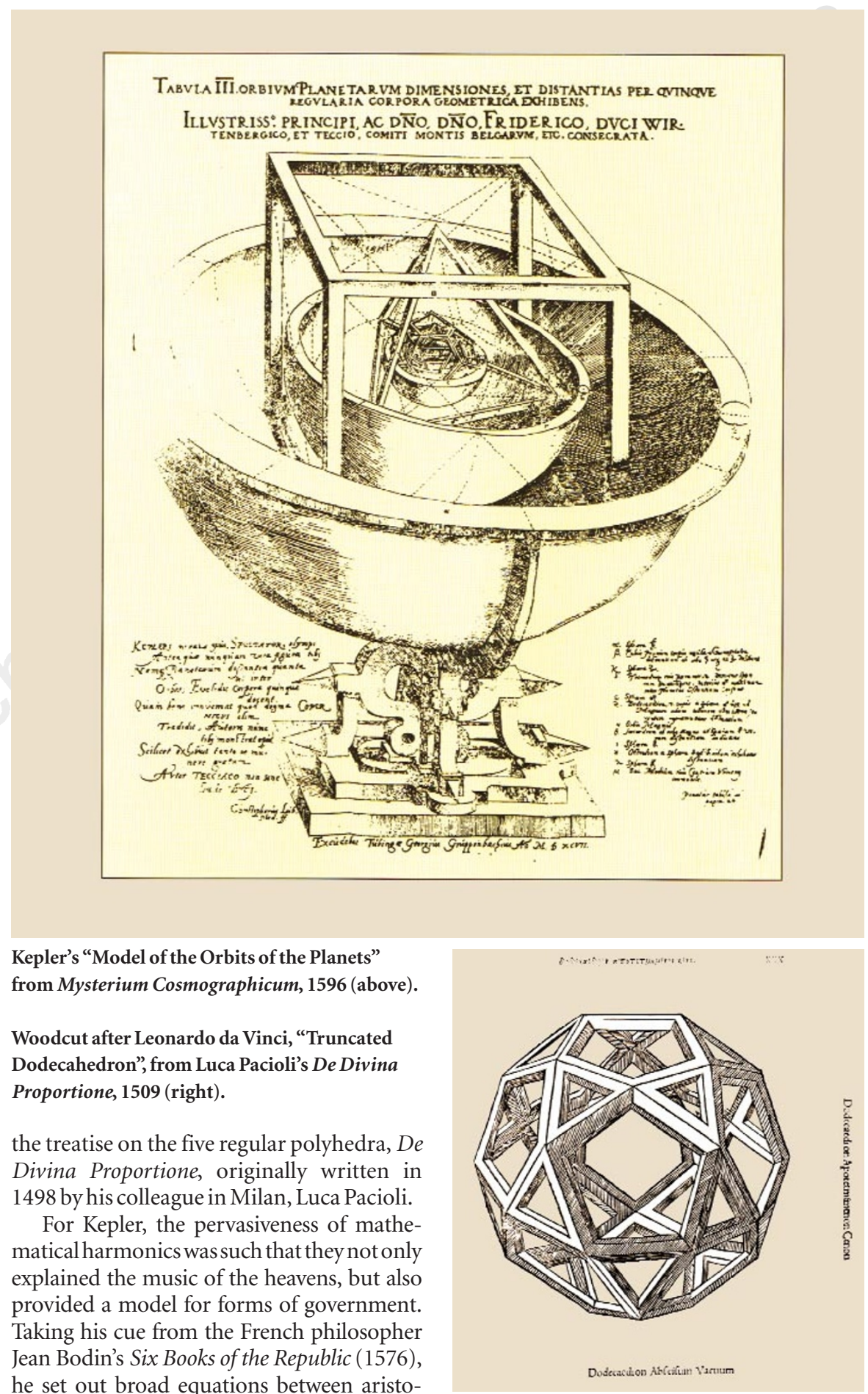

he set out broad equations between aristocratic republics and the kind of proportions expressible in numbers, and between royal governance and the incommensurable properties of geometrical proportions. Kepler argued that the harmony of proportion should ideally permeate every relationship in society from the formulation of laws to the weighting of punishment and from the delights of friendship to the division of inheritances. Tempered proportion and apportionment is the rule in all things. The aim is that things should be on Earth as in the heavens. $\square$ Martin Kemp is in the Department of the History of OX12PG, UK

e-mail:martin.kemp@trinity.oxford.ac.uk Art, University of Oxford, 35 Beaumont Street, Oxford 